\title{
Direct Writing in Polymers with Femtosecond Laser Pulses: Physics and Applications
}

\author{
Kallepalli Lakshmi Narayana Deepak, Venugopal Rao Soma \\ and Narayana Rao Desai
}

Additional information is available at the end of the chapter

http://dx.doi.org/10.5772/45854

\section{Introduction}

Nonlinear optical phenomena in the optical spectral range followed by the invention of laser in early 1960's directed the generation of optical pulses using Q switching and mode locking techniques. Ultrafast lasers with extremely short pulse duration $(<100 \mathrm{fs})$ opened a new avenue towards fabrication of integrated photonic and signal processing devices in a variety of transparent materials. A new approach for the local modification of transparent materials through nonlinear optical processes has been investigated due to extraordinarily high peak intensities of short pulses. A variety of materials including metals, dielectrics, polymers, and semiconductors have been successfully processed by the use of fs pulses [1-14]. Bulk refractive index change in transparent materials is found to be useful in applications of waveguides. Various applications resulting from fs laser writing of different materials, especially in polymers, have been successfully demonstrated in the fields of micro-fluidics, bio-photonics, and photonics etc. [15-32]. The minimal damage arising from the generation of stress waves, thermal conduction, or melting has proved to be one of the main responsible mechanisms for various applications demonstrated using fs laser micromachining. In the present chapter we discuss the formation of free radicals and defects which are responsible for emission in polymer systems. The impact of fs lasers pulses causing minimal damage can be utilized to fabricate emissive micro-craters, especially in polymers. These emissive microcraters find prospective applications in memory based devices.

\section{Experimental}

Herein we present detailed micro-structure fabrication procedures and spectroscopic investigations of those structures in four different polymers (a) Poly Methyl Methacrylate 
(PMMA) (b) Poly Di Methyl Siloxane (PDMS) (c) Polystyrene (PS) and (d) Poly Vinyl Alcohol (PVA). The spectroscopic investigations were carried out in both bulk and thin films of polymers. We used a Ti: Sapphire laser delivering $100 \mathrm{fs}$ pulses at $800 \mathrm{~nm}$ with an energy of $1 \mathrm{~mJ}$ per pulse at $1 \mathrm{kHz}$ repetition rate. The input energy was varied using half wave plate and polarizer combination. Three Newport stages with $15 \mathrm{~nm}$ resolution were arranged three dimensionally and the translation was controlled using computer controlled program. We used 40X and 20X microscope objectives with 0.65 and 0.4 numerical apertures, respectively, to focus the laser beam in our experiments. CCD camera was used while Z stage of the micro-fabrication set up was adjusted for fabricating structures either on the surface or inside the bulk. Figure 1 shows schematic diagram of the micro-fabrication setup. As shown in figure 1 the laser pulses were passed through half wave plate (HWP) and polarizer (BP) so that energy could be varied using this combination. Apertures were introduced in middle to align the beam. M1-M3 are the mirrors used to make the beam incident vertically onto the three dimensionally arranged Newport stages. We fabricated several micro-structures, micro-craters, surface and subsurface diffraction gratings, surface grids, microfluidic channels and arbitrary shapes in these polymers. Since these polymers are transparent and have large band gap, we calculated the Keldysh parameter for these polymers to assess the dominant ionization mechanism. Tunneling ionization was found to be the main responsible mechanism for all the investigated polymers. The spectroscopic investigations were carried out in both bulk and thin films of polymers. Bulk PMMA and PS were purchased from Goodfellow, USA and UK. PVA thin films were prepared by dissolving 8.56 grams of PVA beads in $100 \mathrm{ml}$ of water and thin films were prepared using spin coating technique. Thin films of PS are made by preparing the PS solution first. Solution of PS was prepared by mixing 1 gram of polystyrene beads (ACROS) in $8 \mathrm{ml}$ toluene and stirred for 48 hours for complete miscibility. We prepared thin films of PS on a glass plate by spin coating the solution of PS. All these polymers were cut into $1 \mathrm{~cm} \times 1 \mathrm{~cm}$ square pieces, polished using different grades of polishing sheets and sonicated in distilled water before micro fabrication experiments were carried out.

The energy of an $800 \mathrm{~nm}$ photon corresponds to $1.55 \mathrm{eV}$ while the optical band gap of pure PMMA being $4.58 \mathrm{eV}$ implies that the nonlinear process involving at least three photons is responsible for structural modification at the focal volume [33]. Schaffer et al. [34] have shown that there are three possible mechanisms viz. tunneling, intermediate, and multiphoton ionizations that take place when transparent material interacts with femtosecond pulses. The Keldysh parameter which tells us the mechanism that is dominant is defined as $\gamma=(\omega / \mathrm{e})\left(\mathrm{m} \times \mathrm{c} \times \mathrm{n} \times \varepsilon_{0} \times \mathrm{Eg} / \mathrm{I}\right)$ where ' $\omega$ ' is the laser frequency, ' $\mathrm{I}$ ' is the laser peak intensity at the focus, ' $\mathrm{m}$ ' and ' $\mathrm{e}$ ' are the reduced mass and charge of the electron, respectively, and ' $\mathrm{c}$ ' the velocity of light, $\mathrm{n}$ is the refractive index of the material, $\mathrm{Eg}$ is the band-gap of the material and $\varepsilon_{0}$ is the permittivity of free space. For our studies that Keldysh parameter was $<1.5$ illustrating tunneling as responsible mechanism for structures written using 40X and 20X microscopic objectives. Figure 2 shows the plot obtained for Keldysh parameter with different energies ranging from $150 \mu \mathrm{J}$ to $30 \mathrm{~nJ}$ (equivalent to 83 to $0.017 \mathrm{PW} / \mathrm{cm}^{2}$ ). Results obtained with different polymers using fs laser direct writing are explained in detail in references 35-41. 


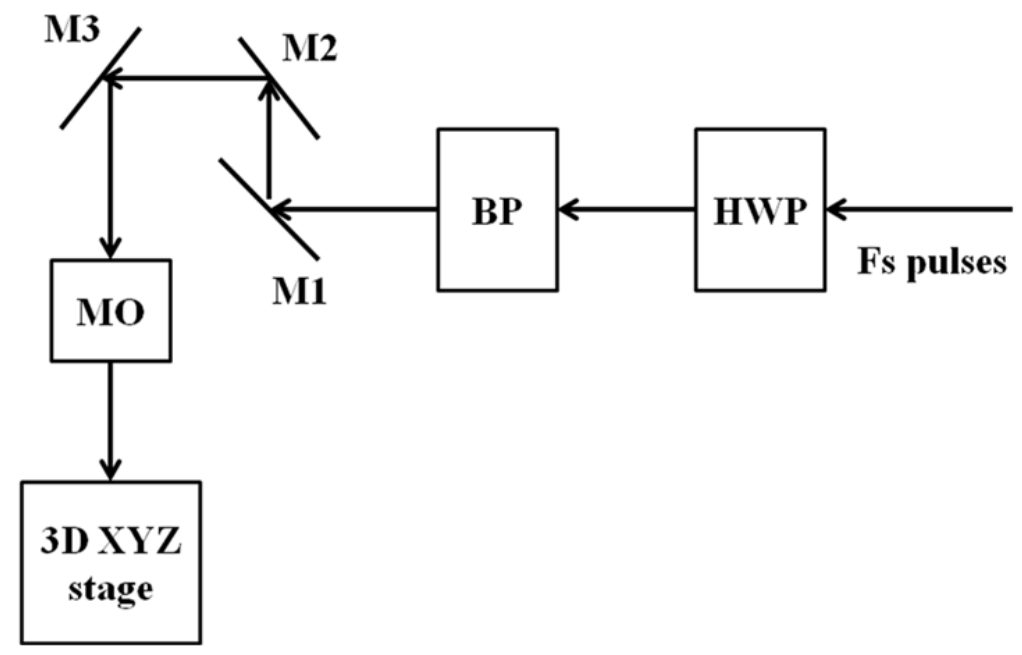

Figure 1. Experimental setup for microfabrication

Our initial studies suggested an increase in structure width with number of scans, energy, and focusing conditions. Figure 3(a) shows some of the Field Emission Scanning Electron Microscope (FESEM) images of the fabricated microstructures on PVA thin film $(60-80 \mu \mathrm{m}$ thickness). We clearly observed the formation of a trough in the central portion of each structure. It is evident as central portion of the incident Gaussian pulse has more intensity (thereby affecting the polymer more) resulting in void formation. This phenomenon was observed for energies ranging from $100 \mu \mathrm{J}$ to $10 \mu \mathrm{J}$. Figure $3(\mathrm{~b})$ shows evidently the formation of trough in the central portion of the microstructure fabricated at $100 \mu \mathrm{J}$ energy with $1 \mathrm{~mm} / \mathrm{s}$ speed. For structures fabricated at low energies (typically less than $10 \mu \mathrm{J}$ ) formation of trough was not observed. Figure 3(c) shows a plot of width of microstructure as well as trough obtained at different energies for different sets. Formation of micro-craters was seen at $1 \mu \mathrm{J}$ energy and with $1 \mathrm{~mm} / \mathrm{s}$ speed. Structures fabricated with 635 and $564 \mathrm{~nJ}$ obviously demonstrated formation of micro-craters. Figure 4(a) shows the structure fabricated at $1 \mu \mathrm{J}$ energy, while $4(\mathrm{~b})$ and 4 (c) show the FESEM images of the microstructures fabricated at 635 and $564 \mathrm{~nJ}$ energies with $1 \mathrm{~mm} / \mathrm{s}$ speed. In our experiments with different polymers we observed that the effect of fs pulses on these polymers, leading to the formation of defects resulting in emission, formation of paramagnetic centers, and broadening of Raman vibrational modes in the fs laser modified regions. Figure 5 shows the part of shape 8 fabricated in PMMA. Since the image was big only a part of 8 shape, which is curved, is shown. We captured the emission from the fs laser modified region using a confocal microscope. We could clearly observe pseudo-green color from the fs laser modified regions which is an indication of emission. Similar results were obtained in case of other polymers also, details of which are summarized in references 35-41. By combining the formation of micro-craters (which took place at high scan speed $>3 \mathrm{~mm} / \mathrm{s}$ and low energies of $\sim \mathrm{nJ}$ ) and the emission coming from the modified regions we establish the possibility of using the fluorescent micro-craters towards memory based applications. 


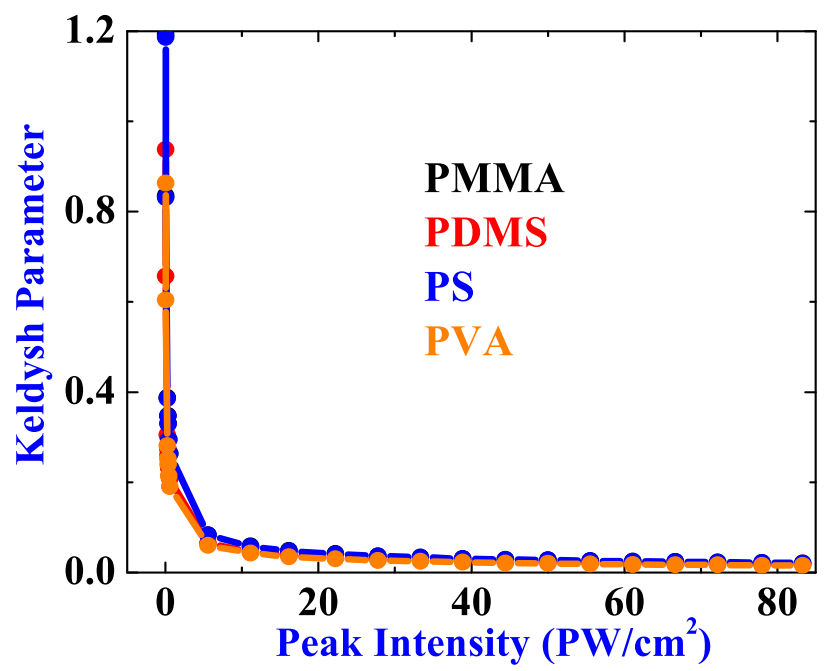

Figure 2. Keldysh parameter of investigated polymers with different peak intensities.

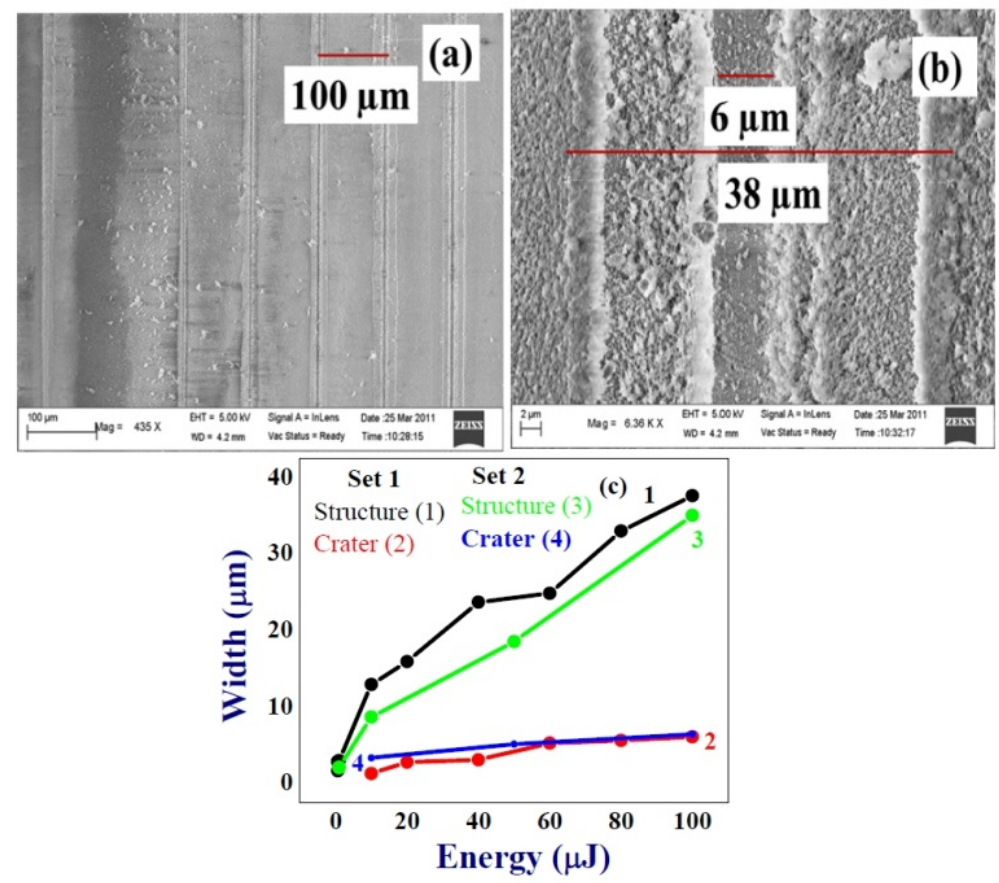

Figure 3. (a) FESEM images of structures fabricated. Structures from right to left were fabricated with energies from 100-10 $\mu \mathrm{J}$ in steps of $20 \mu \mathrm{J}$. (b) Microstructure fabricated at $100 \mu \mathrm{J}$ energy, $1 \mathrm{~mm} / \mathrm{s}$ speed. (c) Plot of structure and trough widths with energy. 


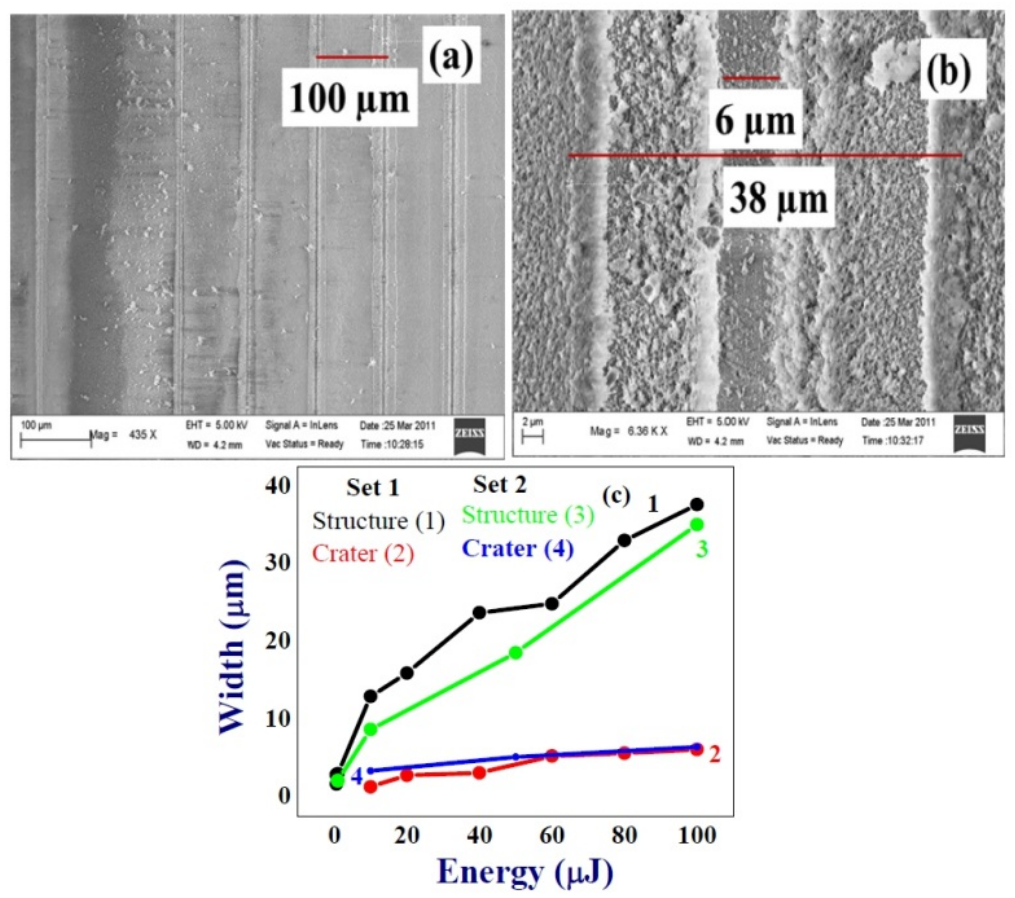

Figure 4. FESEM images microstructure fabricated at $1 \mathrm{~mm} / \mathrm{s}$ speed, with energy of (a) $1 \mu \mathrm{J}$, (b) $635 \mathrm{~nJ}$, and (c) $564 \mathrm{~nJ}$.

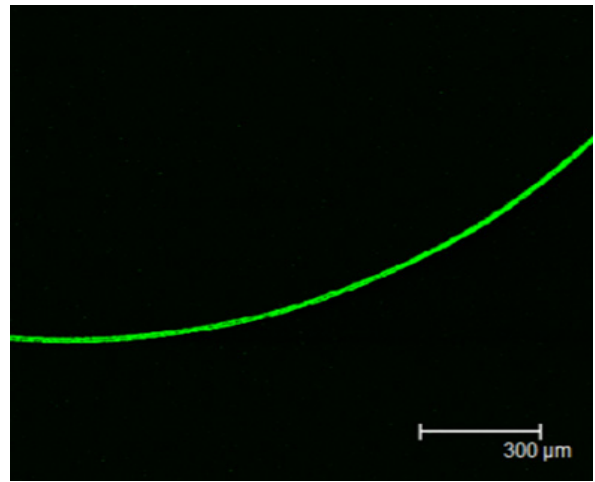

Figure 5. Part of the shape 8 fabricated in PMMA. Pseudo green color shows emission from the modified region excited at $488 \mathrm{~nm}$. Scale bar is $300 \mu \mathrm{m}$.

Structures were also fabricated at different energies $(1 \mu \mathrm{J}, 635 \mathrm{~nJ}, 168 \mathrm{~nJ}$, and $33 \mathrm{~nJ})$ and different scanning speeds to study the formation of micro-craters in PS. Figure 6(a) shows the confocal microscope image of a buried single microstructure in PS fabricated at $1 \mu \mathrm{J}$ 
energy and $1 \mathrm{~mm} / \mathrm{s}$ speed. For the same energy we observed the appearance of craters when the scanning speed was increased to $2 \mathrm{~mm} / \mathrm{s}$. This is depicted clearly in figure $6(\mathrm{~b})$, where the formation of micro-craters was just onset. The process of formation of micro-craters is evident from the edges of figure 6(b). Figures 6(c) and 6(d) illustrate the confocal microscope images of the craters formed at still higher scanning speeds of 3 and $4 \mathrm{~mm} / \mathrm{s}$, respectively. From these data we confirmed that by increasing the scanning speed one can reduce the pulse to pulse overlap and the micro-craters can be obtained similar to observations of periodic refractive index modulation observed earlier in glasses. The same trend was observed even at other energies also. However, we found that the formation of craters at higher scanning speeds and lower energies exhibited better profiles. This is obvious because the intensity of heat waves generated at each position where the pulse impinges depends on the pulse energy.
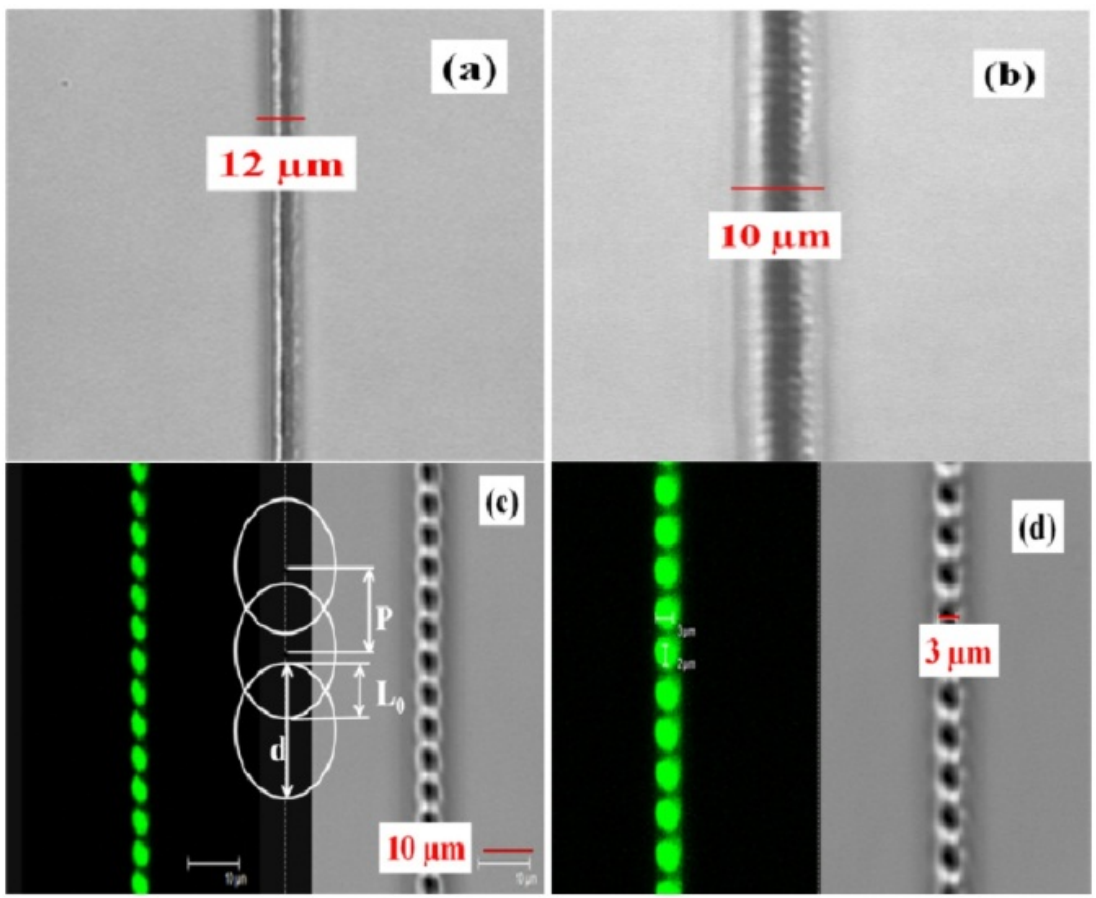

Figure 6. (a) Confocal microscope image of a buried single micro-structure in PS fabricated at $1 \mu \mathrm{J}$ energy and $1 \mathrm{~mm} / \mathrm{s}$ speed. Structure width is $\sim 12 \mu \mathrm{m}$. (b) Beginning of formation of micro- craters at the same energy when scanning speed is increased to $2 \mathrm{~mm} / \mathrm{s}$. Structure width $10 \mu \mathrm{m}$. (c) Scanning speed increased to $3 \mathrm{~mm} / \mathrm{s}$ and the inset shows model used to fabricate micro- craters (d) Confocal microscope image of the craters formed at still higher scanning speed of $4 \mathrm{~mm} / \mathrm{s}$. Pseudo green color indicates emission from the modified micro-craters when excited at $488 \mathrm{~nm}$ wavelength. Crater size is $3 \mu \mathrm{m}$. 


\begin{tabular}{|c|c|c|c|c|}
\hline S. No. & $\begin{array}{c}\text { Energy } \\
\text { (nJ) }\end{array}$ & $\begin{array}{l}\text { Speed } \\
(\mathrm{mm} / \mathrm{s})\end{array}$ & $\begin{array}{l}\text { Observation } \\
\text { (Line/Crater) }\end{array}$ & $\begin{array}{c}\text { Structure/Crater } \\
\text { Size }(\mu \mathrm{m})\end{array}$ \\
\hline \multirow{9}{*}{1} & \multirow{9}{*}{1000} & 1 & Line & 12 \\
\hline & & 1.5 & Line & 10 \\
\hline & & 2 & Line & 10 \\
\hline & & 2.5 & Crater formation started & 6 \\
\hline & & 3 & Crater & 6 \\
\hline & & 3.5 & Crater & 4 \\
\hline & & 4 & Crater & 3 \\
\hline & & 4.5 & Crater & 4 \\
\hline & & 5 & Crater & 3 \\
\hline \multirow{9}{*}{2} & \multirow{9}{*}{635} & 1 & Line & 14 \\
\hline & & 1.5 & Line & 12 \\
\hline & & 2 & Crater formation started & 10 \\
\hline & & 2.5 & Crater & 3 \\
\hline & & 3 & Crater & 3 \\
\hline & & 3.5 & Crater & 3 \\
\hline & & 4 & Crater & 3 \\
\hline & & 4.5 & Crater & 3 \\
\hline & & 5 & Crater & 3 \\
\hline \multirow{9}{*}{3} & \multirow{9}{*}{168} & 1 & Line & 4 \\
\hline & & 1.5 & Crater formation started & 3 \\
\hline & & 2 & Crater & 2 \\
\hline & & 2.5 & Crater & 2 \\
\hline & & 3 & Crater & 2 \\
\hline & & 3.5 & Crater & 2 \\
\hline & & 4 & Crater & 2 \\
\hline & & 4.5 & Crater & 2 \\
\hline & & 5 & Crater & 2 \\
\hline \multirow{9}{*}{4} & \multirow{9}{*}{33} & 1 & Line & 3 \\
\hline & & 1.5 & Crater formation started & 3 \\
\hline & & 2 & Crater & 2 \\
\hline & & 2.5 & Crater & 2 \\
\hline & & 3 & Crater & 2 \\
\hline & & 3.5 & Crater & 2 \\
\hline & & 4 & Crater & 2 \\
\hline & & 4.5 & Crater & 2 \\
\hline & & 5 & Crater & 2 \\
\hline
\end{tabular}

Table 1. Details of micro crater structures fabricated at different energies and scanning speeds. 
Therefore, at higher energies these intense heat waves travel and overlap with the waves generated at other positions. This leads to the formation of a line instead of a crater. This aspect is highlighted in table 1. As energy is reduced, micro-crater formation occurred at lower scan speeds, indicating the fact that the effect of pulse to pulse overlap controlled by scan speed and the phenomena of generation/propagation of heat waves at each position are important factors in the fabrication of micro-craters. Inset of figure 6(c) shows a model that represents the formation of micro-craters. The pitch ' $\mathbf{p}$ ' which is the distance from center to center can be varied by setting the scanning speed according to the relation $\mathbf{p}=\mathbf{s} / \mathbf{f}$ where ' $\mathbf{s}$ ' is scanning speed and ' $\mathbf{f}$ ' is laser repetition frequency, which is $1 \mathrm{kHz}$ in our case. Lo is the overlap region between two consecutive focused spots which is (d-p) where $d$ is the diameter of the spot. The spot size is calculated using the relation $D=1.22 \lambda / \mathrm{NA}$ where $\mathrm{D}$ is the diameter of the focused spot, $\lambda$ is the wavelength and NA is the numerical aperture of the microscope objective used. We had used a 40X microscope objective with NA of 0.65 in our experiments. The estimated spot size was $\sim 1.55 \mu \mathrm{m}$. In our experiments on micro-crater fabrication we observed clear micro-crater formation from $3 \mathrm{~mm} / \mathrm{s}$ speed onward with different energies as tabulated in table 1 . Hence the minimum pitch $(\mathbf{p})$ can be taken as $3 \mu \mathrm{m}$.

As the radius of the focused spot was $\sim 0.75 \mu \mathrm{m}$, the micro craters start appearing for speeds beyond $3 \mathrm{~mm} / \mathrm{s}$. However, we could obtain minimum crater size of $\sim 2 \mu \mathrm{m}$ in our experiments owing to the size of the focused spot and continuous translation. The fabrication of these micro craters is useful in the areas of memory storage devices and photonic crystals.

For better understanding about the emission originating from modified regions, we fabricated microstructures over a large area to record the emission and excitation spectra using conventional fluorescence spectrometer. To accomplish this, an array of closely spaced lines were fabricated in PMMA (energy $10 \mu \mathrm{J}$, speed $1 \mathrm{~mm} / \mathrm{s}$, period $30 \mu \mathrm{m})$, PDMS $(50 \mu \mathrm{J}$, $1 \mathrm{~mm} / \mathrm{s}, 10 \mu \mathrm{m})$, PS $(1 \mu \mathrm{J}, 0.5 \mathrm{~mm} / \mathrm{s}, 30 \mu \mathrm{m})$, and PVA $(10 \mu \mathrm{J}, 1 \mathrm{~mm} / \mathrm{s}, 20 \mathrm{~m})$ using a $40 \mathrm{X}$ objective. All the four polymers are transparent to visible light before fs laser irradiation and hence do not show any emission when excited with visible light. However, due to multiphoton absorption overcoming the large band gaps of these polymers, they get modified and optical centers are produced that exhibit emission when excited at different excitation wavelengths. We observed this kind of emission when the fs laser fabricated channels were irradiated with visible wavelengths such as $458 \mathrm{~nm}, 488 \mathrm{~nm}, 514 \mathrm{~nm}$ wavelengths. The observation of emission and change in emission peak with the excitation wavelength were observed and could be interpreted in the context of formation of myriad optical centers or defects. The increase in emission intensity with irradiation dose in bulk and thin films of polymers are due to the formation of more number of optical centers with irradiation dose.

Nurmukhametov et al. [42] have exposed PS films and solutions with UV laser light beam $(\lambda$ $=248 \mathrm{~nm}$ ) and observed changes in absorption and luminescent properties. They observed formation of optical centers with absorption band from $280 \mathrm{~nm}$ to $460 \mathrm{~nm}$ with fluorescence band from $330 \mathrm{~nm}$ to $520 \mathrm{~nm}$. They established different optical centers being responsible for such a behavior and hypothesized their results more close to the spectrum of 
diphenylbutadiene (DPBD). They figured out three optical centers responsible for the behavior of emission namely trans-stilbene, DPBD and diphenylhexatriene (DPHT). The absorption and emission spectra due to the fs laser irradiation in the present study match nearly with their reported work indicating the creation of similar optical centers. Figures 7(a) - 7(c) show the emission peaks are different for different excitation wavelengths. This emission was recorded using a confocal microscope. We feel that the modification of PS was due to $800 \mathrm{~nm}$ (infrared) laser through multi-photon absorption process. Consequently, fs laser irradiation results in bond scission followed by the formation of large number of defects that act as optical centers for emission. This resulted in shift in emission peak with excitation wavelength. The observed spectral peaks are mentioned in table 2. Observed spectral peaks from fluorescence spectrometer and the confocal microscope are also compared in the table.

\begin{tabular}{|c|c|c|c|}
\hline S. No. & $\begin{array}{c}\text { Excitation } \\
\text { Wavelength } \\
\text { (nm) }\end{array}$ & $\begin{array}{c}\text { Emission peak (nm) } \\
\text { observed in confocal } \\
\text { microscope }\end{array}$ & $\begin{array}{c}\text { Emission peak (nm) } \\
\text { observed in fluorescence } \\
\text { spectrometer }\end{array}$ \\
\hline 1 & 458 & 509,544 & 505,536 \\
\hline 2 & 488 & 535 & 550 \\
\hline 3 & 514 & 565 & 563 \\
\hline 4 & 400 & $\begin{array}{c}\text { Excitation source was not } \\
\text { available }\end{array}$ & $454,462,476$ \\
\hline
\end{tabular}

Table 2. Comparison of emission data obtained in irradiated PS (PSG1).
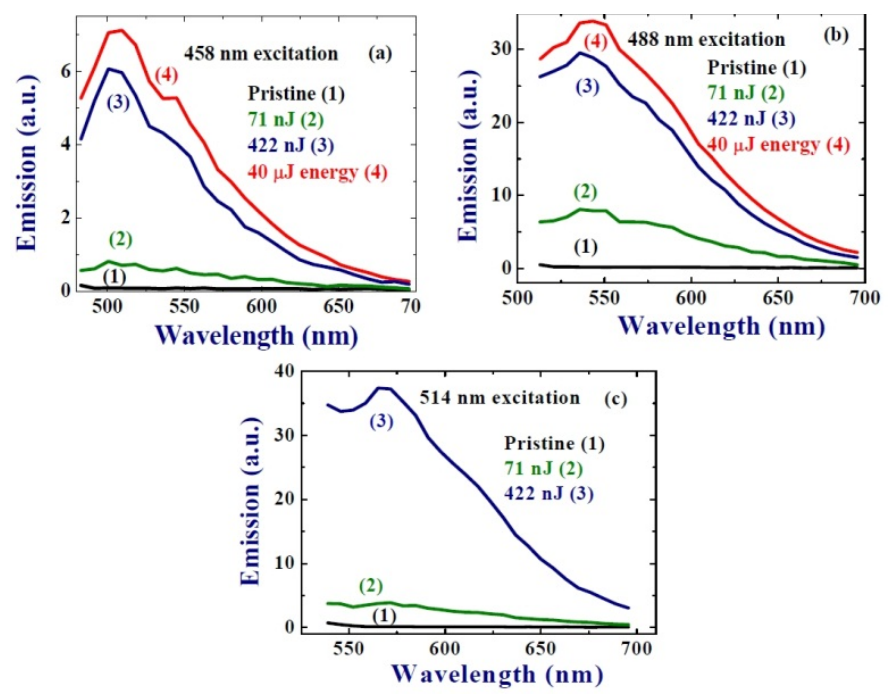

Figure 7. (a) Plots of emission when structures fabricated at $40 \mu \mathrm{J}, 422 \mathrm{~nJ}, 63 \mathrm{~nJ}$ and pristine region of PS excited at $458 \mathrm{~nm}$; (b) Plots of emission when structures fabricated at $40 \mu \mathrm{J}, 422 \mathrm{~nJ}, 63 \mathrm{~nJ}$ and pristine region of PS excited at $488 \mathrm{~nm}$; (c) Plots of emission when structures fabricated at $422 \mathrm{~nJ}, 63 \mathrm{~nJ}$ and pristine region of PS excited at $514 \mathrm{~nm}$. 
The effect of change in emission peak with excitation wavelength was observed in all polymers and is attributed to the Red Edge Effect (REE). The change in emission peak with excitation wavelength in case of PS is shown in figure 8(a). The excitation spectra collected at different monitoring wavelengths looked alike which is depicted in figure 8(b). Inset of figure 8(b) shows the transmission spectra of un-irradiated and irradiated PS. Varieties of fluoropores in different media with frozen or relatively slow structural dynamics from vitrified and highly viscous solutions to polymer matrices have shown similar effects. These phenomena did not account for concepts of independence of emission energy on excitation energy within the absorption band (Vavilov's law) and the occurrence of emission irrespective of excitation band, always from the lowest electronic and vibrational state of same multiplicity called Kasha's Rule [43-46]. These effects originate not from the violation of fundamental principles but from their operation in specific conditions when the ensemble of excited molecules is distributed in interaction energy with molecules in their surroundings. In a condensed medium, this distribution always exists at the time of excitation, but its display in a variety of spectroscopic phenomena depends on how fast the transitions are between the species forming this ensemble of states. Thus, REE is a site-selective effect which allows probing of the dynamics of redistribution of fluoropores between different environments.
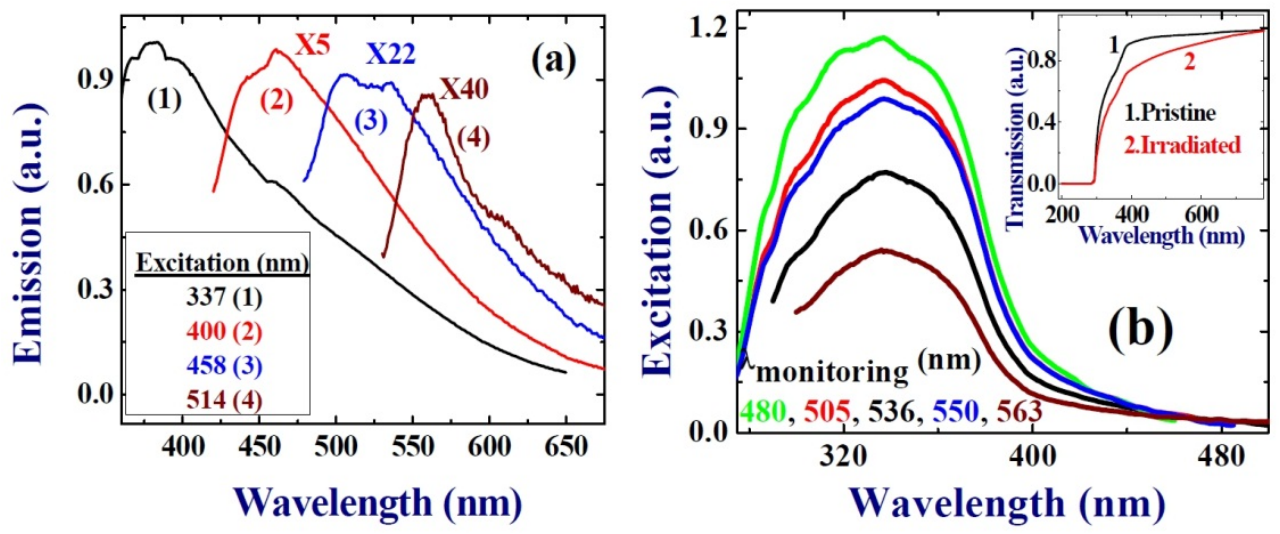

Figure 8. (a) Emission from PSG1 grating excited at different wavelengths. Emissions with longer wavelength excitations are enhanced for clarity (b) Excitation spectra of irradiated PS at different monitoring wavelengths. Inset of (b) shows the transmission spectra of unirradiated and irradiated PS.

The absorption characteristics of these optical centers thus formed showed maximum absorption at same wavelength. As the defect centers are distributed over wide range of energies, the emission spectrum too gets distributed over the entire fluorescence spectrum contribution coming from all types of defects. This results in the spectra being the same in the excitation spectra recorded by monitoring different emission wavelengths. However the emission, when excited at long wavelength side, due to localization of energy we observe different emission spectra. Figure 9(a) shows the plot of excitation wavelength with the observed emission wavelength for all polymers which is an indication of REE effect. Since these polymers contain active functional groups such as aldehyde, ketone etc., the 
absorption maximum within these functional groups can also lead to the observed emission. In the case of PMMA, the maximum excitation near $370 \mathrm{~nm}$ can be ascribed to $\mathrm{n} \rightarrow \pi^{*}$ transition. In case of PS due to the presence of ring, it could be due to $\pi \rightarrow \pi^{*}$ transition. After these functional groups are excited they can get de-excited to any of the electronic states by emission process. Figure 9(b) shows a schematic diagram for the functional groups within these polymers undergoing different transitions. We also investigated the role of possible vibrational levels in emission spectra we recorded. For this, we excited all irradiated polymers from $250 \mathrm{~nm}-560 \mathrm{~nm}$ in the range of $20 \mathrm{~nm}$ and collected emission spectra. Interestingly, some of the emission peaks coincided with Raman peaks of these polymers. Plots 10 (a)-(b) show the emission spectra recorded for fs laser irradiated PMMA at different excitation wavelengths. The energy difference between the excitation and the emission peak is calculated in the wave numbers using the relation $\Delta \mathrm{E} / \mathrm{hc}\left(\mathrm{cm}^{-1}\right)=1 / \lambda_{\mathrm{ext}}-1 / \lambda_{\mathrm{em}}$, where $\lambda_{\text {ext }}$ and $\lambda_{\mathrm{em}}$ are excitation and emission wavelengths respectively. For PMMA, the emission peaks due to excitation wavelengths at $458,488,530$, and $560 \mathrm{~nm}$ nearly match the Raman mode of characteristic peak at $1736 \mathrm{~cm}^{-1}$ of $\gamma(\mathrm{C}=\mathrm{O})$ of $(\mathrm{C}-\mathrm{COO})$ mode [47]. Excitations at 380, and $400 \mathrm{~nm}$ nearly match with Raman mode of $3454 \mathrm{~cm}^{-1}$ which is $2 \gamma_{2}$ overtone of $1730 \mathrm{~cm}^{-1}$. Emission peak at $543 \mathrm{~nm}$ matched with another Raman peak of combination band involving $\gamma(\mathrm{C}=\mathrm{C})$ and $\gamma(\mathrm{C}-\mathrm{COO})$ of PMMA. The spectra therefore contain the peaks due to transition from the excited state to the vibrational states. The shift in the emission peaks with excitation wavelength is shown in figure 10 (b). This shift in the peak of the emission with excitation could be due to the excitation of different localized states created during laser irradiation. Table 3 shows excitation, emission peaks and the calculated energy difference between the excitation and the emission peaks.
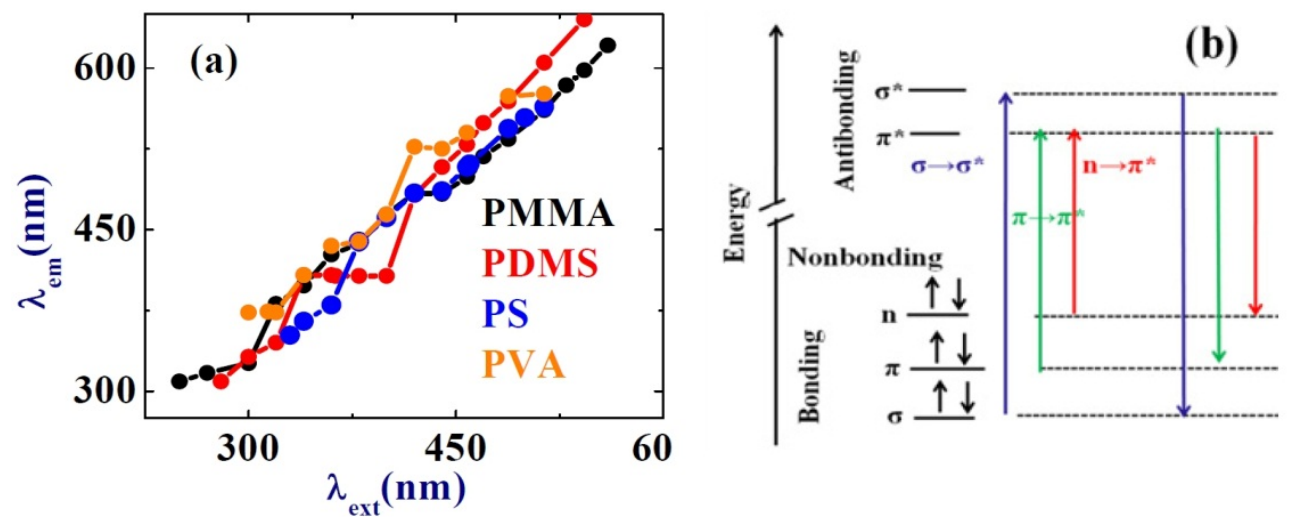

Figure 9. (a) REE in investigated polymers (b) Energy level diagram.

Different research groups have worked on electron spin resonance (ESR) of polymers [48-50]. However, reports on ESR analysis of fs laser irradiated polymers are sparse as the field has gained momentum recently and many theoretical and experimental results need to be explored further. In our endeavor towards understanding ESR of fs irradiated polymers, we fabricated two dimensional grids to increase effective area of irradiation for ESR analysis. We observed peroxide type free radicals when these polymers were treated with fs laser. Pure polymers such 
as PMMA are not paramagnetic substances and hence do not contain any paramagnetic centers. So, there were no peaks observed in ESR spectrum. When polymers PMMA, PDMS, and PS are treated with fs laser, they showed ESR signal which is an indication of existence of peroxide type free radicals. Figure 11 shows ESR signal in PS. In case of PS, the radicals are alkoxy radicals as reported in literature [51]. Further, confocal micro-Raman studies were carried out for fabricated structures in the channels. Formation of defects such as optical centers and free radicals led to broadening of Raman peaks and reduction in Raman intensity due to high intense shock waves formed at the center of the Gaussian pulse. In order to study the local effect, a microstructure was fabricated on a PVA thin film at two different energies $(635 \mathrm{~nJ}$ and $10 \mu \mathrm{J}$ ) with speed of $1 \mathrm{~mm} / \mathrm{s}$. Figure 12 shows the Raman spectra collected in the middle and end regions of the structures fabricated. Raman spectra recorded for higher energies showed the broadening of Raman modes along with decrease in Raman intensity compared with structures fabricated at low energies and pristine regions of the polymer from which we could conclude the formation of defects. We observed similar effects even in other polymers also.
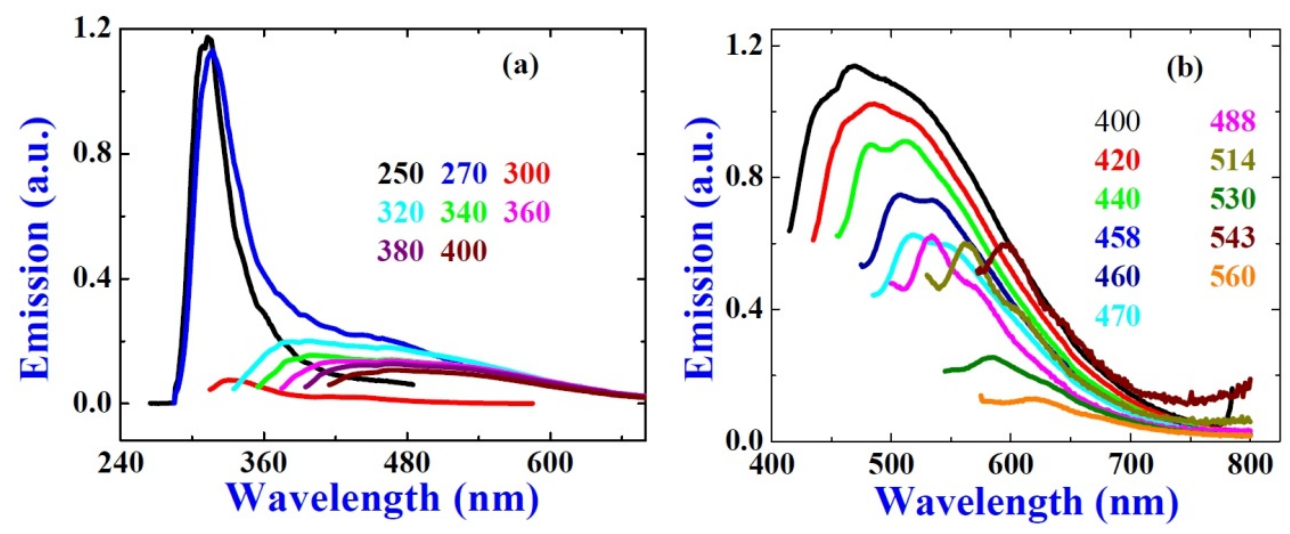

Figure 10. Plot of emission spectra of (a) PMMA at excitation wavelengths from 250-400 nm (b) PMMA at excitation wavelengths from $400-560 \mathrm{~nm}$

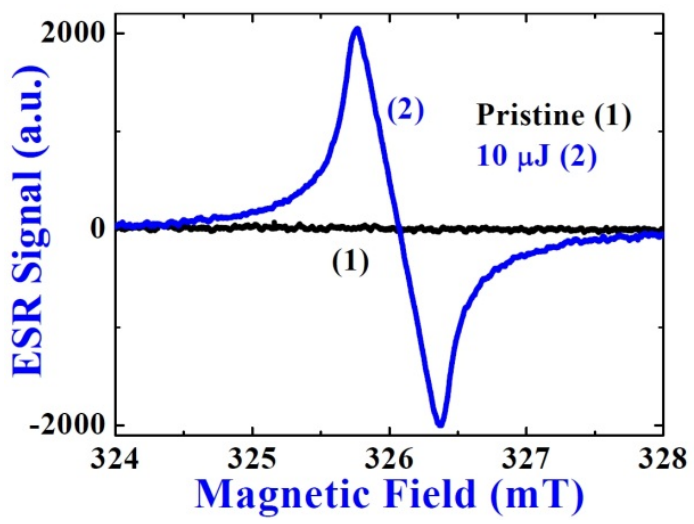

Figure 11. ESR signal observed in irradiated PS. 


\begin{tabular}{|c|c|c|c|}
\hline S. No. & $\lambda_{\text {ext }}(\mathrm{nm})$ & $\lambda_{\mathrm{em}}(\mathrm{nm})$ & $\Delta \mathrm{E} / \mathrm{hc}\left(\mathrm{cm}^{-1}\right)=\lambda_{\mathrm{ext}^{-1}}-\lambda_{\mathrm{em}^{-1}}$ \\
\hline \multirow{2}{*}{1} & \multirow{2}{*}{250} & 309 & 7638 \\
\hline & & 313 & 8051 \\
\hline 2 & 270 & 317 & 5491 \\
\hline 3 & 300 & 326 & 2658 \\
\hline \multirow{3}{*}{4} & \multirow{3}{*}{320} & 381 & 5003 \\
\hline & & 398 & 6214 \\
\hline & & 461 & 9558 \\
\hline \multirow{2}{*}{5} & \multirow{2}{*}{340} & 398 & 4286 \\
\hline & & 464 & 7860 \\
\hline \multirow{2}{*}{6} & \multirow{2}{*}{360} & 427 & 4359 \\
\hline & & 465 & 6272 \\
\hline \multirow{2}{*}{7} & \multirow{2}{*}{380} & 437 & 3432 \\
\hline & & 467 & 4903 \\
\hline 8 & 400 & 464 & 3448 \\
\hline 9 & 420 & 484 & 3148 \\
\hline \multirow{2}{*}{10} & \multirow{2}{*}{440} & 483 & 2023 \\
\hline & & 511 & 3158 \\
\hline \multirow{2}{*}{11} & \multirow{2}{*}{458} & 499 & 1794 \\
\hline & & 529 & 2930 \\
\hline \multirow{2}{*}{12} & \multirow{2}{*}{460} & 507 & 2015 \\
\hline & & 535 & 3048 \\
\hline \multirow{2}{*}{13} & \multirow{2}{*}{470} & 518 & 1972 \\
\hline & & 551 & 3128 \\
\hline \multirow{2}{*}{14} & \multirow{2}{*}{488} & 534 & 1765 \\
\hline & & 568 & 2886 \\
\hline 15 & 514 & 561 & 1630 \\
\hline 16 & 530 & 584 & 1745 \\
\hline 17 & 543 & 598 & 1694 \\
\hline 18 & 560 & 621 & 1754 \\
\hline
\end{tabular}

Table 3. Excitation, emission, and Raman shift observed in fs laser irradiated PMMA.

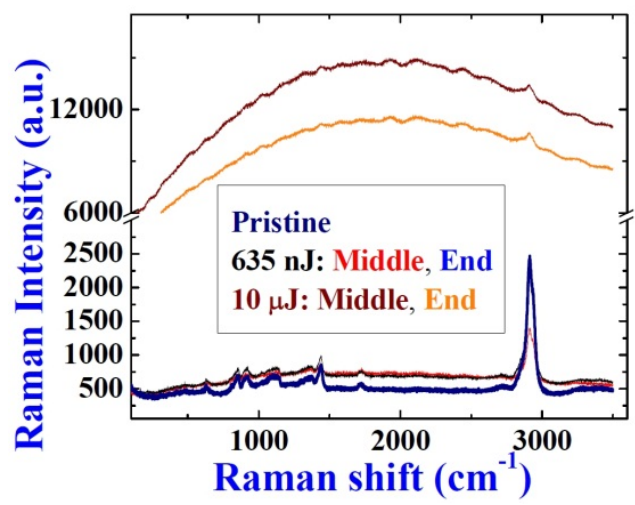

Figure 12. Confocal Raman spectra for structures fabricated on PVA thin film. 


\section{Conclusions}

We presented our results on the physical and spectroscopic investigations of fs laser irradiated polymers. The unusual behavior of emission observed in case of these polymers is attributed to the red edge effect. The transitions involved with in the functional groups such as $n \rightarrow \pi^{*}$, and $\pi \rightarrow \pi^{*}$ are responsible for the emission observed. The role of coincidence of Raman signals with emission observed is illustrated. The presence of paramagnetic centers is confirmed through ESR studies. Both the defects which are responsible for emission and ESR signal are further confirmed through confocal micro Raman studies by observing the broadening of Raman modes.

\section{Author details}

Kallepalli Lakshmi Narayana Deepak

School of Physics, University of Hyderabad, Gachibowli, Hyderabad, India

Laboratoire LP3, UMR 6182, CNRS-Universite Aix-Marseille, Pole Scientifique et Technologique de Luminy, Case 917, Marseille, France

Soma Venugopal Rao

Advanced Center for Research in High Energy Materials (ACRHEM), University of Hyderabad,

Gachibowli, Hyderabad, India

Desai Narayana Rao*

School of Physics, University of Hyderabad, Gachibowli, Hyderabad, India

\section{Acknowledgement}

We are extremely grateful to DRDO, India for all the financial support during the tenure of this project. K. L. N. Deepak acknowledges CSIR for financial support to carry out his doctoral work. DNR and SVR acknowledge Department of Science and Technology (DST), India for financial support through a project SR/S2/LOP-11/2005.

\section{References}

[1] Gattass R R, Mazur E (2008) Femtosecond laser micromachining in transparent materials. Nat. Phot. 2: 219-225.

[2] Juodkazis S, Mizeikis V, Misawa H (2009) Three-dimensional Microfabrication of materials by femtosecond lasers for photonics applications. J. Appl. Phys. 106: 051101.

[3] Nolte S, Will M, Burghoff J, Tuennermann A (2003) Femtosecond waveguide writing: a new avenue to three-dimensional integrated optics. Appl. Phys. A 77: 109-111.

\footnotetext{
${ }^{*}$ Corresponding Author
} 
[4] Ams M, Marshall G D, Dekker P, Dubov, Mezentsev V K, Bennion I, Withford M J (2008) Investigation of ultrafast laser-photonic material interactions: challenges for directly written glass photonics. IEEE J. Sel. Top. Quant. Electron., 14(5): 1370-1381.

[5] Qiu J, Miura K, Hirao K (2008) Femtosecond laser-induced micro features in glasses and their applications. Journal of Non-Crystalline Solids. 354: 1100-1111.

[6] Della Valle G, Osellame R, Laporta P (2009) Micromachining of photonic devices by femtosecond laser pulses. J. Opt. A: Pure Appl. Opt. 11: 013001.

[7] Qiu J, Miura K, Hirao K (2008) Femtosecond laser-induced micro features in glasses and their applications," Journal of Non-Crystalline Solids. 354, 1100-1111, 2008.

[8] Della Valle G, Osellame R, Laporta P (2009) Micromachining of photonic devices by femtosecond laser pulses. J. Opt. A: Pure Appl. Opt. 11: 013001-.

[9] Taccheo S, Della Valle G, Osellame R, Cerullo G, Chiodo N, Laporta P, Svelto O, Killi A, Morgner U, Lederer M, Kopf D (2004) Er : Yb-doped waveguide laser fabricated by femtosecond laser pulses. Opt. Lett. 29: 2626-2628.

[10] Florea C, Winick K A (2003) Fabrication and characterization of photonic devices directly written in glass using femtosecond laser pulses. Journal of Lightwave Technology 21: 246-253.

[11] Minoshima K, Kowalevicz A M, Ippen E P, Fujimoto J G (2002) Fabrication of coupled mode photonic devices in glass by nonlinear femtosecond laser materials processing. Optics Express 10: 645-652.

[12] Streltsov A M, Borrelli N F (2001) Fabrication and analysis of a directional coupler written in glass by nanojoule femtosecond laser pulses. Optics Letters 26: 42-43.

[13] Davis K M, Miura K, Sugimoto N, Hirao K (1996) Writing Waveguides in Glass with a Femtosecond Laser. Optics Letters 21: 1729-1731.

[14] Glezer E N, Milosavljevic M, Huang L, Finlay R J, Her T H, Callan J P, Mazur E (1996) Three-dimensional optical storage inside transparent materials. Opt. Lett. 21(24): 20232025.

[15] Watanabe W, Sowa S, Tamaki T, Itoh K, Nishii J (2006) Three-Dimensional Waveguides Fabricated in Poly(methyl methacrylate) by a Femtosecond Laser. Jap. J. Appl. Phys., Part 2, 45: L675-L767.

[16] Zoubir A, Lopez C, Richardson M, Richardson K (2004) Femtosecond laser fabrication of tubular waveguides in poly(methyl methacrylate). Opt. Lett. 29(16): 1840-1842.

[17] Sowa S, Tamaki T, Itoh K, Nishii J, Watanabe W (2006) Three-dimensional waveguides fabricated in poly(methyl methacrylate) by a femtosecond laser. Jap. J. Appl. Phys., Part 2: Letters 45: 29-32, L765-L767.

[18] Wang K, Klimov D, Kolber Z (2007) Waveguide fabrication in PMMA using a modified cavity femtosecond oscillator. Proc. SPIE 6766: 67660Q.

[19] Watanabe W, Sowa S, Tamaki T, Itoh K, Nishii J(2006) Three-Dimensional Waveguides Fabricated in Poly(methyl methacrylate) by a Femtosecond Laser. Jap. J. Appl. Phys., Part 2, 45: L675-L767. 
[20] Zoubir A, Lopez C, Richardson M, Richardson K (2004) Femtosecond laser fabrication of tubular waveguides in poly (methyl methacrylate). Opt. Lett. 29(16): 1840-1842.

[21] Sowa S, Tamaki T, Itoh K, Nishii J, Watanabe W (2006) Three-dimensional waveguides fabricated in poly(methyl methacrylate) by a femtosecond laser. Jap. J. Appl. Phys., Part 2: Letters 45: 29-32, L765-L767.

[22] Cumpston B H, Ananthavel S P, Barlow S, Dyer D L, Ehrlich J E, Erskine L L, Heikal A A, Kuebler S M, Lee I Y S, McCord-Maughon D, Qin J Q, Rockel H, Rumi M, Wu X L, Marder S R, Perry J W (1999) Two-photon polymerization initiators for three dimensional optical data storage and microfabrication. Nature 398: 51-54.

[23] Nie Z, Lee H, Yoo H, Lee Y, Kim Y, Lim K S, Lee M (2009) Multilayered optical bit memory with a high signal-to-noise ratio in fluorescent polymethylmethacrylate. Appl. Phys. Lett. 94: 111912.

[24] Farson D F, Choi H W, Lu C, Lee L J (2006) Femtosecond laser bulk micromachining of microfluidic channels in poly (methyl methacrylate). J. Laser Appl. 18(3): 210-215.

[25] Day D, Gu M (2005) Microchannel fabrication in PMMA based on localized heating by nanojoule high repetition rate femtosecond pulses. Opt. Exp. 13(16): 5939-5946.

[26] Ding L, Blackwell R I, Kunzler J F, Knox W H (2008) Femtosecond laser micromachining of waveguides in silicone-based hydrogel polymers. Appl. Opt. 47(17): 3100-3108.

[27] Si J, Meng Z, Kanehira S, Qiu J, Hua B, Hirao K (2004) Multiphoton-induced periodic microstructures inside bulk azodye-doped polymers by multibeam laser interference. Chem. Phys. Lett. 399: 276-279.

[28] Si J H, Qiu J R, Zhai J F, Shen Y Q, Hirao K (2002) Photoinduced permanent gratings inside bulk azodye-doped polymers by the coherent field of a femtosecond laser. Appl. Phys. Lett. 80(3): 359-361.

[29] Kim T N, Campbell K, Groisman A, Kleinfeld D, Schaffer C B (2005) Femtosecond laserdrilled capillary integrated into a microfluidic device. Appl. Phys. Lett. 86: 201106.

[30] Mendonca C R, Cerami L R, Shih, Tilghman R W, Baldacchini T, Mazur E (2008) Femtosecond laser waveguide micromachining of PMMA films with azoaromatic chromophores. Opt. Exp. 16(1): 200-206.

[31] Zhou G, Ventura M J, Vanner M R, Gu M (2004) Use of ultrafast-laser-driven microexplosion for fabricating three-dimensional void-based diamond-lattice photonic crystals in a solid polymer material. Opt. Lett. 29: 2240-2242.

[32] Nolte S, Will M, Burghoff J, Tuennermann A (2003) Femtosecond waveguide writing: a new avenue to three-dimensional integrated optics. Appl. Phys. A 77: 109-111.

[33] Baum A, Scully P J, Perrie W, Jones D, Issac R, Jaroszynski D A (2008) Pulse-duration dependency of femtosecond laser refractive index modification in poly (methyl methacrylate). Opt. Lett. 33(7): 651-653.

[34] Schaffer C B, Brodeur A, Mazur E (2001) Laser-induced breakdown and damage in bulk transparent materials induced by tightly focused femtosecond laser pulses. Meas. Sci. Technol. 12: 1784-1794. 
[35] Deepak K L N, Narayana Rao D, Venugopal Rao S (2009) Fabrication and Optical Characterization of microstructures in PMMA and PDMS using femtosecond pulses for photonic and micro fluidic applications. Appl. Opt. 49 (13): 2475.

[36] Deepak K L N, Venugopal Rao S, Narayana Rao D (2010) Femtosecond laser-fabricated microstructures in bulk poly(methylmethacrylate) and poly(dimethylsiloxane) at 800 nm towards lab-on-a-chip applications. Pramana 75(6): 1221-1232.

[37] Deepak K L N, Kuladeep R, Venugopal Rao S, Narayana Rao D (2011) Luminescent microstructures in bulk and thin films of PMMA, PDMS, PVA, and PS fabricated using femtosecond direct writing technique. Chem. Phys. Lett. 503: 57-60.

[38] Deepak K L N, Kuladeep R, Narayana Rao D (2011) Emission properties of femtosecond (fs) laser fabricated microstructures in Polystyrene (PS). Opt. Commun. 284: 3070-3073.

[39] Deepak K L N, Kuladeep R, Praveen Kumar V, Venugopal Rao S, Narayana Rao D (2011) Spectroscopic investigations of femtosecond laser irradiated Polystyrene and fabrication of microstructures. Opt. Commun. 284: 3074-3078.

[40] Deepak K L N, Kuladeep R, Venugopal Rao S, Narayana Rao D (2012) Studies on Defect Formation in Femtosecond Laser Irradiated PMMA and PDMS," Radiation Effects and Defects in Solids 67: 88-101.

[41] Deepak K L N, Venugopal Rao S, Narayana Rao D (2011) Effect of heat treatment to efficient buried diffraction gratings in Polystyrene. Appl. Surf. Sci. 257: 9299-9305.

[42] Nurmukhametov R N, Volkova L V, Kabanov S P (2006) Fluorescence and absorption of polystyrene exposed to UV laser radiation. J. Appl. Spectr. 73: 55-60.

[43] Galley W C, Purkey R M, Role of Heterogeneity of the Solvation Site in Electronic Spectra in Solution, Proc. Natl Acad. Sci. USA 1970; 67, 1116-1121.

[44] Rubinov A N, Tomin VI (1970) Bathochromic luminescence of organic dyes, Opt. Spektr. 29: 1082-1086.

[45] Terenin A N, Fotonika molekul krasitelei, Photonics of Dye Molecules (1967) Nauka:Leningrad, 616.

[46] Birks JB. Photophysics of Aromatic molecules. Wiley-Inter-science: London 1970.

[47] Thomas K J, Sheeba M, Nampoori V P N, Vallabhan C P G, and Radhakrishnan P (2008) Raman spectra of polymethyl methacrylate optical fibres excited by a $532 \mathrm{~nm}$ diode pumped solid state laser.J. Opt. A: Pure Appl. Opt. 10: 055303-055307.

[48] Velter-Stefanescu M, Duliua O G, Preda N (2005) On the relaxation mechanisms of some radiation induced free radicals in polymers. J. Optoelectron. Advanced Mat. 7(2): 985-989.

[49] Kaptan H Y, Tatar L (1997) An Electron Spin Resonance study of mechanical fracture of poly (methyl methacrylate). J. Appl. Polym. Sci. 65: 1161-1167.

[50] Abdelaziz M (2008) Electron spin resonance and optical studies of poly (methylmethacrylate) doped with CuCl2. J. Appl. Polym. Sci. 108: 1013-1020. 
[51] Ohnishi S, Tanei T, Nitta I (1962) ESR Study of Free Radicals Produced by Irradiation in Benzene and Its Derivatives. J. Chem. Phys. 37: 2402-2407. 